\section{US and UK sign Science and Technology Agreement}

$\mathrm{S}$ cience and technology collaboration between the United States (US) and the United Kingdom (UK) received a government-backed boost in September 2017 with the signing of the umbrella Science and Technology Agreement between the two nations. Signed by Judith Garber, US Acting Assistant Secretary of State for Oceans and International Environmental and Scientific Affairs, and Jo Johnson, UK Minister of State for Universities, Science, Research and Innovation, the agreement outlines "a commitment to collaborate on world-class science and innovation," according to a joint statement by the US and UK governments.

While this is called the first formal science and technology (S\&T) agreement between the US and the UK, the joint statement characterizes the ongoing scientific partnership between these two nations as "one of the world's strongest" and notes that it has already resulted in 26 Nobel prizes across science and economics. In addition, an umbrella agreement for S\&T cooperation between the US and the EU-of which the UK is currently a member - was signed in 1997 and has been extended to October 2018. But with the 2016 referendum decision to leave the EU, the UK has begun to pursue its own international cooperation and policy agreements. data will be shared.
The text of the S\&T agreement is not yet publicly available, but both the US and UK governments have released some details. According to the White House Office of Science and Technology Policy (OSTP), the agreement is meant to cover a range of science and policy areas, including "basic science, early-stage R\&D, emerging technologies like artificial intelligence, promising new public-private partnership models, and the role that science and technological advancement play in economic prosperity." The OSTP also says the agreement establishes a guide for cooperation that details how intellectual property resulting from US-UK collaboration will be handled and how expertise, materials, equipment, and

The first major project of the S\&T agreement is already under way with a UK government investment of $£ 65$ million (approximately USD\$88 million) to help construct the Long-Baseline Neutrino Facility/Deep Underground Neutrino Experiment(LBNF/DUNE). Hosted by the US Department of Energy (DOE) Fermi National Accelerator Laboratory (Fermilab) in Illinois, the LBNF/DUNE project will include a new linear accelerator and an upgrade to Fermilab's existing accelerators to send a beam of neutrinos over 800 miles through the earth to a detector that is being constructed one mile below ground at the

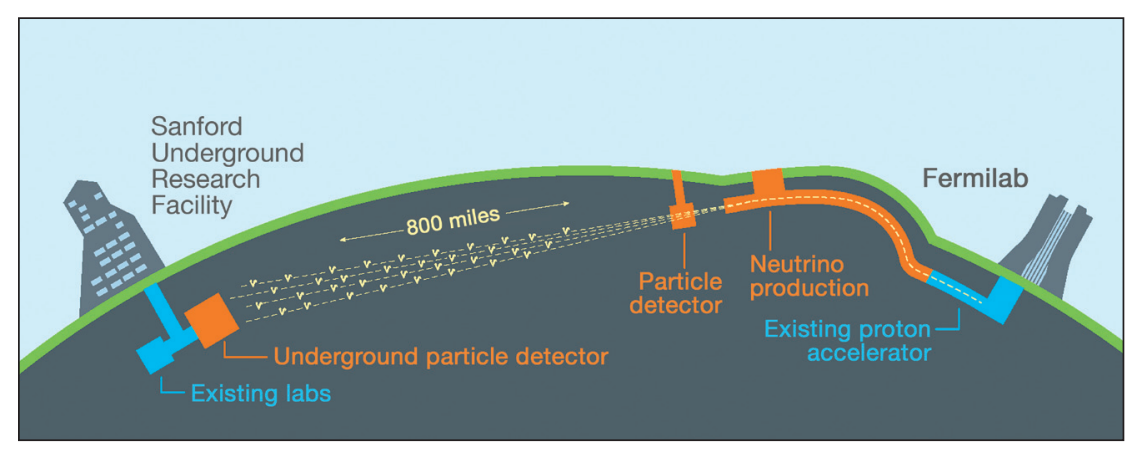

Graphic of the Long-Baseline Neutrino Facility/Deep Underground Neutrino Experiment (LBNF/DUNE) project. Credit: Fermilab.
Sanford Underground Research Facility in South Dakota. According to a UK government press release on the S\&T agreement, "the UK is a major scientific contributor to the DUNE collaboration, with 14 UK universities and two Science and Technology Facilities Council laboratories providing essential expertise and components to the experiment and facility."

While the US, UK, and CERN are the primary investors in the LBNF/DUNE project, it is supported by 31 countries represented by over 1000 scientists from more than 170 laboratories and universities. "The scale of the LBNF/DUNE project is massive, and formalizing an agreement like this is important to facilitate investment and collaboration," says Nigel Lockyer, Fermilab Director. In addition to the scale, Lockyer explains that the LBNF/ DUNE project does not follow the typical formula for US science facilities and experiments and is the first of its kind in the US:

The LBNF/DUNE project is the first fully international mega-science project hosted by the US that has been collaborative from the beginning. In the past, DOE has built facilities and then invited international collaboration on experiments. But with the LBNF/DUNE project, both the facility and the experiment have been fully collaborative from the beginning. Everyone who is a part of the project has a seat at the table, and experts from around the world inform the entire project, from construction of the facility to design of the experiments that will be conducted. It follows the model used by CERN where the world came together to study the Higgs Bosonnow the world is coming together to study the neutrino at LBNF/DUNE.

The goal of the LBNF/DUNE project is to study the behavior of neutrinos and antineutrinos to better understand the so-called "big bang" (the explosion that created the universe) and the resulting structure of the universe, including why matter dominated over antimatter. It will also explore cosmic events like supernovae by detecting neutrinos that are expelled when stars explode. 
Lockyer likened the neutrino/antineutrino experiment to a sort of x-ray that would essentially allow scientists and researchers to "look inside the explosion" and test theories about the origins of the universe. And in addition to expanding the frontiers of physics, Lockyer points out that there are a number of technical challenges associated with a project like this and says that "one of the side benefits is the development of other areas of science to enable these large-scale experiments." With regard to materials science, Lockyer highlighted ongoing research into the response of target materials to increasingly powerful doses of radiation. This could lead to the discovery of new materials or materials properties that could be useful in space and medical applications where radiation plays a significant role.

A second project supported under this agreement is a collaborative effort between the US Smithsonian Institution and the UK Arts and Humanities Research Council to enhance digital research at museums. While exhibitions take center stage at most museums, behind the scenes, these exhibitions are enhanced and supported by researchers and scientists. In fact, the
Smithsonian's National Museum of American History, where the US-UK digital research project will be based, employs more than 200 scientists across a spectrum of research areas. The digital research project aims to improve data analysis and define best practices in using digital technologies for research. In addition, the project includes plans to deploy digital technologies at museums, which is expected to "further audience engagement" according to the UK government press release, and improve science, technology, engineering, and mathematics (STEM) education programs, according to the OSTP.

Despite the fact that the agreement is not yet publicly available and no projects aside from the two just highlighted have been announced, it is highly likely that many areas of research_-including materials research-will be covered. The OSTP has listed development of MRI and PET standards, quantum technologies, and collaborations on autonomous transportation technologies as being considered under the agreement. R\&D of quantum technologies, in specific, will certainly rely on the rapidly growing field of quantum materials research.
"From infrared detectors, sensors, and low noise amplifiers, to low power logic and quantum computation, there are a number of areas that the US and UK governments can benefit from technologies that utilize quantum phenomenon," says Javad Shabani, assistant professor of physics at the Center for Quantum Phenomena at New York University. Shabani also points out that collaborating with the UK on quantum materials and technologies makes sense because the UK "has a strong background in materials synthesis and coating, which has led to great success stories in the development of compound semiconductors, phase-change materials, graphene, and high-temperature superconductivity.'

The full scope of the US-UK S\&T agreement and its impacts on collaboration, science funding, and innovation remain to be seen, but both nations appear optimistic. The joint press release says that the cooperation on science and technology should help both nations maintain global leadership in research, which has the "potential to be world-changing."

Jennifer A. Nekuda Malik
OPEN SESAME brings training to students from the Middle East www.opensesame-h2020.eu

$\mathrm{F}_{\mathrm{f}}$ ollowing an open call, nine students from SESAME members have been awarded training fellowships to work in European light source laboratories in 2018. OPEN SESAME is a Horizon 2020 project, which runs until the end of 2019. It provides training opportunities for the SESAME light source in Jordan. An intergovernmental organization, SESAME's members are Cyprus, Egypt, Iran, Israel, Jordan, Pakistan, the Palestinian Authority, and Turkey. This call for fellowships was open to students working toward master's or doctoral degrees in the realm of light source science in any of these members.

Close to 50 applications were received. After scrutiny by an expert committee, nine places were offered, with six candidates in reserve. The successful applicants represent four SESAME members, with two coming from Egypt and two from Iran, four from Pakistan, and one from Turkey. Seven are women and two are men, and each will be spending a minimum of eight weeks between February and June 2018 in European laboratories. Their fields of interest are all areas that will be addressed by SESAME's phase-one beamlines, namely powder diffraction, x-ray absorption spectroscopy, infrared microspectroscopy, macromolecular crystallography, and $\mathrm{x}$-ray tomography. These techniques address questions ranging from life sciences where antibiotic resistance in bacteria and the interactions of essential oils and macromolecules will be investigated by single-crystal diffraction, to geology where oil and gas flow properties in porous rock will be characterized by hard x-ray microtomography.

The laboratories hosting the fellowships are ALBA in Barcelona, which will host four students; Elettra in Trieste, which will host one; ESRF in Grenoble hosting two; and SOLEIL, near Paris, which will also host two. The Fellows each receive a stipend of $€ 6600$ to cover their costs.

OPEN SESAME is coordinated by ESRF and involves the light source laboratories ALBA, DESY, Elettra, SESAME, and SOLEIL, as well as CERN, which has a long-standing link to SESAME, the French CNRS' Ancient Materials Research Platform, Ipanema, the Cyprus Institute, the European Research Infrastructure Consortium, Instruct, and Italy's INFN. OPEN SESAME's overall objective is to support the initial training needs of SESAME and thereby help the laboratory become established as an integral part of the global light source research landscape. 